\title{
Postinhibitory rebound delay and weak synchronization in Hodgkin-Huxley neuronal networks
}

\author{
David T. W. Chik and Z. D. Wang \\ Department of Physics, The University of Hong Kong, Pokfulam Road, Hong Kong, China
}

(Received 4 April 2003; published 12 September 2003)

\begin{abstract}
Noise-induced weak synchronized oscillatory activities in a globally inhibitory coupled Hodgkin-Huxley neuronal network are studied numerically. A kind of intrinsic delay induced by the postinhibitory rebound is observed and is found to be important in determining the overall frequency of the network. Synchronization occurs in an optimal range of noise intensity with a bell-shaped curve when the inhibitory coupling strength is sufficiently strong. Comparisons with the results for the excitatory coupling are also addressed.
\end{abstract}

DOI: 10.1103/PhysRevE.68.031907

PACS number(s): 87.18.Sn, 87.19.La, 05.40.-a

For the past many years, the stabilities of synchrony, clustering, and other global patterns have been extensively studied in the framework of the nonlinear dynamics of coupled neural oscillators [1,2]. Remarkably, synchronized oscillatory activities, such as those occurring in the mammalian visual cortex during perception [3] and in human thalamocortical area during dream state [4], have been observed in various nervous systems. Interestingly, many regions that perform synchronized oscillatory activities are found to involve inhibitory neurons [5]. According to conventional wisdom, the role of inhibitory neurons in a network is believed to be suppressing each other and generating a winner-take-all competition [6]. In order to have more free neurons to survive, this kind of network is seldomly coupled in an all-to-all structure, which allows more information to be stored. Apart from the alive-or-dead pattern, nevertheless, global synchronization can also occur, provided that delays exist among neurons. So far, two types of delay in the neural network, i.e., the transmission delay and the synaptic delay, have been paid considerable attention [7-9]. The transmission delay corresponds to the time needed for the action potential to travel in the axon or dendrite, which is usually assumed to be a function of distance, or simply a random constant with a certain distribution [7]. The synaptic delay corresponds to the rise and decay time of the synaptic current, which depends on the chemical property of the synapse and is a function of the presynaptic potential $[8,9]$. These two kinds of delay exist in both excitatory and inhibitory networks.

To our understanding, there also exist a third type of delay caused by postinhibitory rebound (PIR) (also known as anode break excitation), which has received less attention in the past. This type of delay exists only in inhibitory networks. It was found that some types of neurons can fire a rebounded spike after inputting a negative current pulse, which was observed experimentally in neocortical neurons [10] and could be simulated mathematically with the Hodgkin-Huxley ( $\mathrm{HH}$ ) model [11] (to be shown later). Note that in the $\mathrm{HH}$ model, both sodium and potassium currents contribute to the occurrence of PIR, while in the other model for thalamus [12], the PIR is based on the slow calcium currents. Although these two models have relevance to different biological systems, the mechanisms of PIR appear to be similar.

On the other hand, the PIR delay is different from the transmission and synaptic delays mainly in that it is an in- trinsic dynamical property of the membrane rather than an independent explicit parameter. The phenomenon of PIR was believed to be important in some biological functions such as auditory coding [13] and central pattern generator [14]. In a central pattern generator, for example, very strong inhibitory couplings are found in the crossed interneurons that produce alternating rhythms through PIR. We think that PIR may also play an important role in synchronization in, for example, neocortex. Previous studies indicated that in the presence of an external delay (either transmission delay or synaptic delay), synchronization occurs in a globally coupled inhibitory network (see Refs. [7] and [9]). In this paper, we find that the PIR delay is also able to induce the global synchronization even in the absence of external delay. In particular, we identify an intrinsic source of delay to be the finite response time of the system dynamics and elucidate its role in the determination of the global timing of the network.

Let us consider a globally connected neuronal network with the Hodgkin-Huxley model, which is described by the following equations:

$$
\begin{gathered}
\frac{d V_{i}}{d t}=f_{i}\left(V_{i}, m_{i}, n_{i}, h_{i}\right)+I_{i}-\eta_{i}-\frac{1}{N-1} \\
\times \sum_{j=1, j \neq i}^{j=N}\left(V_{i}-V_{s y n}\right) J_{i j} G_{j}, \\
\frac{d m_{i}}{d t}=\frac{m_{\infty}(V)-m_{i}}{\tau_{m}(V)}, \\
\frac{d n_{i}}{d t}=\frac{n_{\infty}(V)-n_{i}}{\tau_{n}(V)}, \\
\frac{d h_{i}}{d t}=\frac{h_{\infty}(V)-h_{i}}{\tau_{h}(V)},
\end{gathered}
$$

where

$$
\begin{aligned}
f_{i}\left(V_{i}, m_{i}, n_{i}, h_{i}\right)= & -g_{N a} m_{i}^{3} h_{i}\left(V_{i}-V_{N a}\right)-g_{K} n_{i}^{4}\left(V_{i}-V_{K}\right) \\
& -g_{L}\left(V_{i}-V_{L}\right) .
\end{aligned}
$$

Here, $V_{i}$ is the membrane potential of the $i$ th neuron, $m_{i}$ and $h_{i}$ the activation and inactivation variables of sodium current, and $n_{i}$ the activation variable of potassium current. The 
parameters $g_{N a}, g_{K}$, and $g_{L}$ are the maximum conductances per surface unit for the sodium, potassium, and leak currents, while $V_{N a}, V_{K}$, and $V_{L}$ are the corresponding reversal potentials. $m_{\infty}, h_{\infty}, n_{\infty}$ represent the saturation values, and $\tau_{m}$, $\tau_{n}, \tau_{h}$ represent the relaxation times of the gating variables. Detailed values of these parameters can be found in Ref. [11]. $I_{i}$ is a weak constant current input to the $i$ th neuron which is set at $6.0 \mu \mathrm{A} / \mathrm{cm}^{2}$ for any $i$; as a result, all neurons are biased near the threshold of saddle-node bifurcation such that an extra input can excite the neurons and produce action potentials. The coupling strength between the $i$ th and $j$ th neurons is denoted as $J_{i j} . V_{s y n}$ is the synaptic resting potential: $V_{s y n}=0$ for excitatory coupling with $J_{i j}=J_{e x}$ and $V_{s y n}$ $=-80 \mathrm{mV}$ for inhibitory coupling with $J_{i j}=J_{i n} . G_{j}$ represents the synaptic current, which is commonly modeled by an $\alpha$ function [8] that can be described by other two differential equations:

$$
\begin{aligned}
& \frac{d G_{j}}{d t}=\frac{1}{\tau_{s y n}}\left(-G_{j}+H_{j}\right), \\
& \frac{d H_{j}}{d t}=\frac{1}{\tau_{s y n}}\left(-H_{j}+\Theta_{j}\right),
\end{aligned}
$$

with $\Theta_{j}$ being a step function and equals 1 when the $j$ th neuron fires, or 0 otherwise. $\tau_{s y n}$ is the time constant of the postsynaptic potential, which is also referred to as the synaptic delay. There is another delay term, transmission delay $\Delta$, which does not appear explicitly in the above equations. When the presynaptic neuron fires, the synaptic current will reach the postsynaptic neuron after time $\Delta$. There are $N$ neurons in the network. Each neuron is subject to an independent noise $\eta_{i}$ with the same intensity. The noise undergoes an Ornstein-Uhlenbeck process $\tau_{c}\left(d \eta_{i} / d t\right)=-\eta_{i}+\sqrt{2 D} \xi$, where $\xi$ is Gaussian white noise, $D$ is intensity, and $\tau_{c}$ $=0.1 \mathrm{msec}$ is the correlation time of the noise [15]. The whole set of equations is evaluated numerically using fourthorder Runge-Kutta method with time step $=0.01 \mathrm{msec}$.

We plot the time series of the membrane potential of an $\mathrm{HH}$ neuron for both negative and positive input pulses in Fig. 1(a). For a negative input (solid line), the membrane potential is recovered after the pulse ends, and one rebounded spike is produced at time around $7.5 \mathrm{msec}$. For a positive input (dotted line), the membrane potential rises immediately from the input time of the pulse. A spike is produced with the peak at about $0.1 \mathrm{msec}$. Inside the network, a neuron will receive a positive (or negative) pulse from the presynaptic neuron if the synaptic coupling is excitatory (or inhibitory). We observe that for excitatory coupling there is almost no delay, while for inhibitory coupling there is a delay. This delay is produced by the rebound mechanism, which is a result of the different dynamical fixed points of the ionic currents in the $\mathrm{HH}$ model. Under the influence of a negative input, the membrane potential reaches a new stable value with a reduced outward potassium current and an increased inward sodium current, causing an overall ionic influx. When the input ends, the recovering ionic currents force the mem-

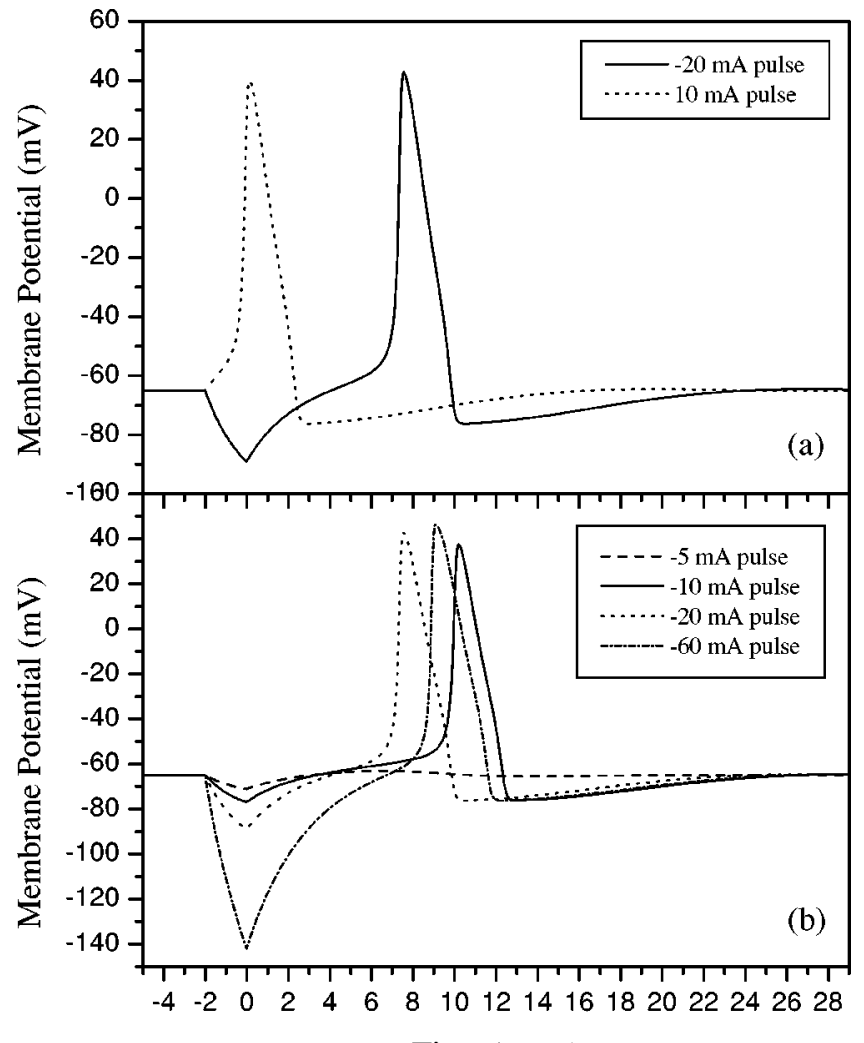

Time (msec)

FIG. 1. The time series of the membrane potential of a HodgkinHuxley neuron. A square current pulse is input from time -2 to 0 msec. The amplitudes of the pulses are (a) $-20 \mathrm{~mA}$ for the solid line and $10 \mathrm{~mA}$ for the dotted line; (b) $-5 \mathrm{~mA}$ for the dashed line, $-10 \mathrm{~mA}$ for the solid line, $-20 \mathrm{~mA}$ for the dotted line, and $-60 \mathrm{~mA}$ for the dash-dotted line.

brane potential to follow a spikelike dynamical trajectory before returning to the original fixed point (resting potential). In Fig. 1(b), we show the influences of the negative pulse strength on the PIR delay. There is a threshold for the PIR. If the negative pulse is weaker than the threshold (for example, $-5 \mathrm{~mA}$ ), the potential simply returns to rest and no spike will be produced (dashed line). If it is just stronger than the threshold of rebound (for example, $-10 \mathrm{~mA}$ ), the rebounding time will be very long (solid line). If it is even stronger (for example, $-20 \mathrm{~mA}$ ), the delay will be shorter (dotted line). If it is very strong (for example, $-60 \mathrm{~mA}$ ), the rebounding time will be longer again (dash-dotted line). Therefore a minimum delay time can be tuned by the pulse strength [16]. On the other hand, the duration of the pulse can also affect the length of PIR delay (not shown here). If the current pulse is longer, the rebound will be faster, and vice versa. In addition, the constant bias also has an effect. The higher the positive subthreshold constant bias is, the shorter the PIR delay, and vice versa. The conclusion is that the length of the delay varies in a complex way with both the amplitude and the duration of the input pulse, as well as the constant bias of the neuron.

Next, we study the condition of coupling strength $J$ for the occurrence of synchronization. Table I shows whether the synchronization occurs (Yes) or not (No) for different ranges 
TABLE I. Occurrence of synchronization.

\begin{tabular}{|c|c|c|}
\hline & \multicolumn{2}{|c|}{ Synchronization (Yes) or not (No) } \\
\hline & Superthreshold; $D=0$ & Subthreshold; optimal $D$ \\
\hline$J_{e x}>J_{C}$ & Yes & Yes \\
\hline$J_{C}>J_{e x}>0$ & Yes & No \\
\hline$J=0$ & No & No \\
\hline$J_{C}^{\prime}>J_{i n}>0$ & Yes & No \\
\hline$J_{i n}>J_{C}^{\prime}$ & Yes & Yes \\
\hline
\end{tabular}

of coupling strength $J$. The left column refers to the superthreshold situation, in which a steady limit cycle of firing is seen when the constant input $I_{0}$ is above the threshold current $I_{C}>0$ (or the postinhibitory rebound will occur when $I_{0}$ is below the other threshold current $I_{C}^{\prime}<0$ ) in the absence of noise. Correspondingly, there are coupling thresholds $J_{e x}$ $=J_{C}$ and $J_{i n}=J_{C}^{\prime}$ for excitatory and inhibitory cases, respectively. Synchronization occurs when $J$ is nonzero. When $J$ is zero, the neurons are independent of each other since the initial phases are set to be different for each neuron. When the coupling is slightly positive (that is, $J_{C}>J_{e x}>0$ ), the neurons organize slowly and become sychronized into one group after a transition period. This is because the positive coupling acts as a positive feedback in the network system. When $J_{e x}>J_{C}$, not only positive feedback exists but also there is a direct spike formation by the large synaptic current from the strong coupling. Synchronization also occurs when $J_{i n}>0$. A similar positive feedback exists for $J_{C}^{\prime}>J_{i n}>0$, plus a direct spike formation if $J_{i n}>J_{C}^{\prime}$. Although the synaptic current becomes negative, its role of organizing the neurons remains unchanged. However, the neurons do not synchronize into one group, but form two to three clusters. Therefore, the collective spiking behaviors for cases $J_{e x}>0$ and $J_{i n}>0$ are not the same.

The right column refers to the situation where $I_{0}$ is of subthreshold. A small value $\delta$ is deducted from the critical value $I_{C}\left(\delta=0.5 \mu \mathrm{A} / \mathrm{cm}^{2}\right.$ in our simulation). An optimal level of noise exists so that spiking occurs through the mechanism of coherence resonance (CR). Here, the so-called $\mathrm{CR}$ is a phenomenon of noise-induced temporal regularity in dynamical systems [17-19]. In excitable systems, a subthreshold constant bias together with a zero-mean noise can produce a noisy limit cycle (see Fig. 2 in Ref. [18]). An eigenfrequency can be observed through a triangular peak in the power spectrum (see Fig. 3 in Ref. [18]). In this case, we find that synchronization does not occur for $J_{C}>J_{e x}>0$ and $J_{C}^{\prime}>J_{\text {in }}>0$ for any intensity of noise. This is because the positive feedback is insufficient to withstand the diffusive effect of noise. Only when $J_{e x}$ or $J_{i n}$ is stronger than the corresponding threshold will synchronization occur. Initially the neurons fire at different phases, and occasionally a large proportion of neurons may fire within a small time bin. Then the large synaptic current produced by the strong coupling generates a spike in the postsynaptic neuron. This direct spike formation can overcome the diffusive effect of noise because the refractory period after spike formation acts as an extra clock that helps to synchronize the neurons.
In Fig. 2, we plot the rastergrams of a neural network with 300 neurons for a variety of cases. For a specific neuron at a certain time, a dot is plotted if the neuron fires. Suppose each neuron receives an independent noise with the intensity $D$ $=4$ and spikings are caused by CR. For $J=0$, there is no synchronization because of random initial phases and the Gaussian noise inputs [Fig. 2(a)]. The lower panel is a histogram calculating the amount of spikes in different time bins, which is referred to as the global firing histogram. Since there is no synchronization, the global firing histogram shows a fluctuating pattern. For $J_{e x}=10$, raster stripes indicating global synchronized oscillatory activities can be observed [Fig. 2(b)], which is similar to that observed in Ref. [19]. There is a stochastic synchronization with about $95 \%$ neurons of the network fire during a global period. The frequency of the global firing is about $60 \mathrm{~Hz}$, which is the same as the natural CR frequency of individual neurons. The periodic oscillation of individual neurons is induced by the mechanism of CR, and synchronization is induced by a positive feedback mechanism produced by excitatory couplings in the network. Peaks are seen in the global firing histogram [lower panel of Fig. 2(b)]. When a transmission delay $\Delta$ $=7 \mathrm{msec}$ is added for the case $J_{e x}=10$, a kind of weak synchronization is seen [Fig. 2(c)]. For $J_{i n}=30$, a similar weak stochastic synchronization is also seen [Fig. 2(d)]. The similarity between Figs. 2(c) and 2(d) is an evidence for the existence of intrinsic delay in the inhibitory network. For all cases, the subthreshold constant bias $I=6 \mu \mathrm{A} / \mathrm{cm}^{2}$, the synaptic delay $\tau_{s y n}$ is set at 0.01 (which is negligible in our consideration), and the transmission delay $\Delta=0$ except for the case shown in Fig. 2(c).

There are two differences between weak [Figs. 2(c) and 2(d)] and strong synchronization [Fig. 2(b)]. First, the frequency of the global firing pattern for the weak synchronization is higher than that for the strong one, therefore the raster stripes in Figs. 2(c) and 2(d) are denser than those in Fig. 2(b) [20]. Second, there are fewer neurons firing during a global period for the weak synchronization. If we look at the global firing histograms, only about $30-40 \%$ neurons fire during the global period. However, it is inappropiate to describe them as clusters as compared with Ref. [2]. Since each neuron receives an independent noise, their behaviors are also independent. The firing group contains entirely different members each time, and the overall behavior exhibits a weak synchronization of the whole network.

An individual neuron does not fire at every global period, which can be seen in the interspike interval histogram (Fig. 3). With the same condition as that of Fig. 2(d), the firing period of a neuron is found to be about $2,3,4, \ldots$ times of the global period, which means that the neuron skips a random number of global firing cycles. Also the peaks are broad and not exactly located at the multiples of the global period value. Therefore the resulting global synchronization pattern is in fact composed of very stochastic individual behaviors.

We study the power spectra of individual neuron inside the network in Fig. 4. The conditions of Figs. 4(a)-4(d) correspond to those of Figs. 2(a)-2(d) one by one. In the absence of coupling [Fig. 4(a)], a broad peak of about $60 \mathrm{~Hz}$ is seen in the power spectrum, which corresponds to the noisy 


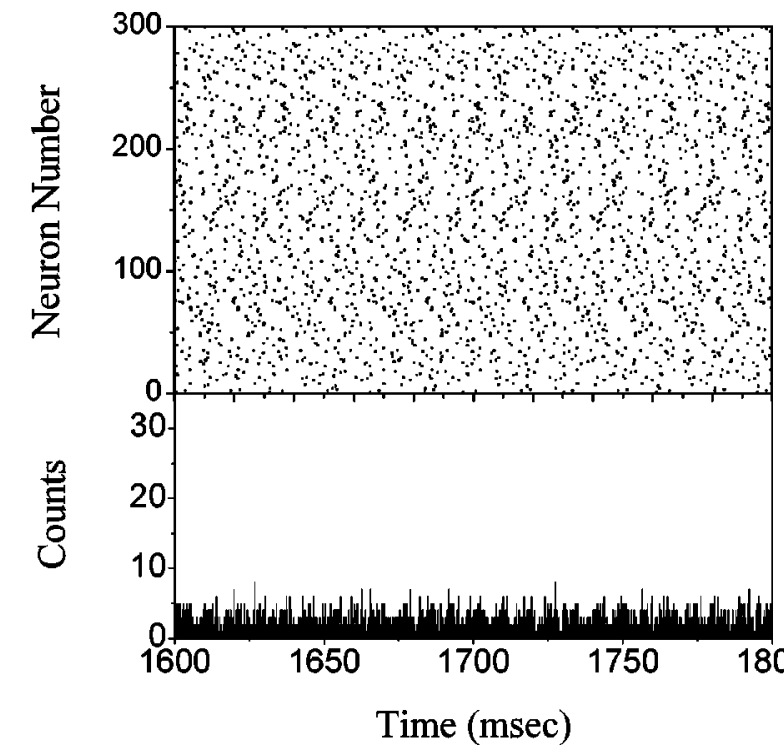

(a)

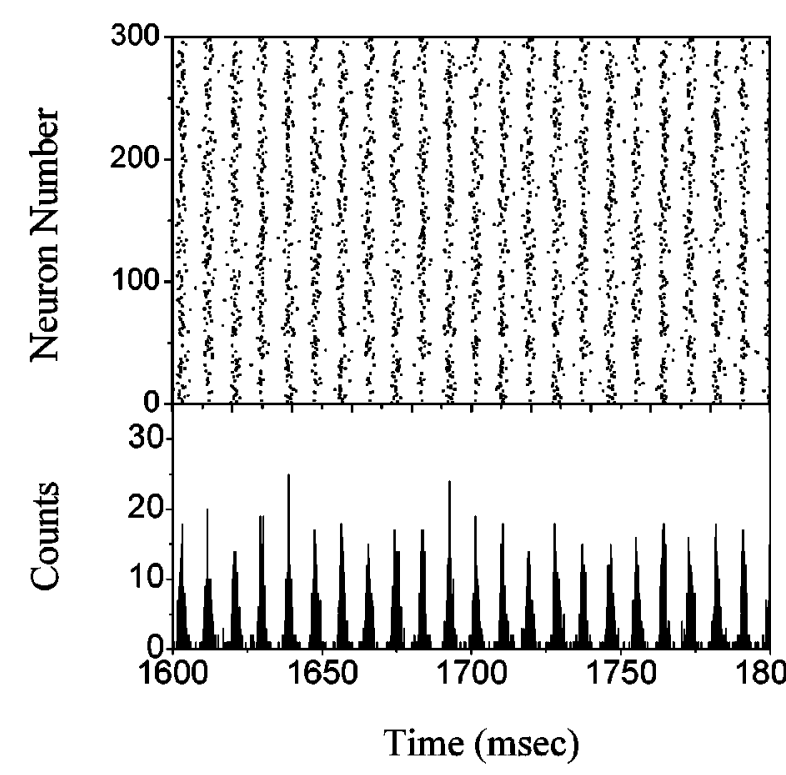

(c)

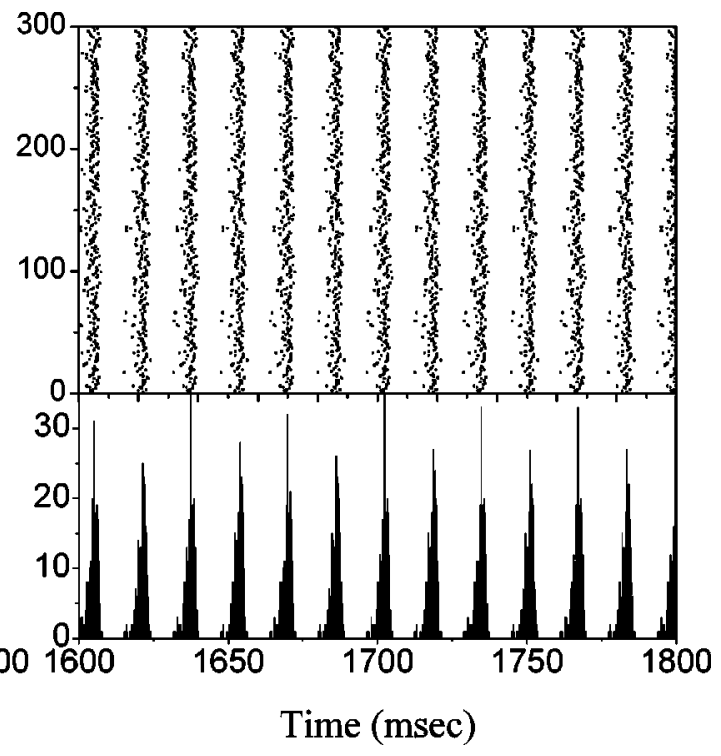

(b)

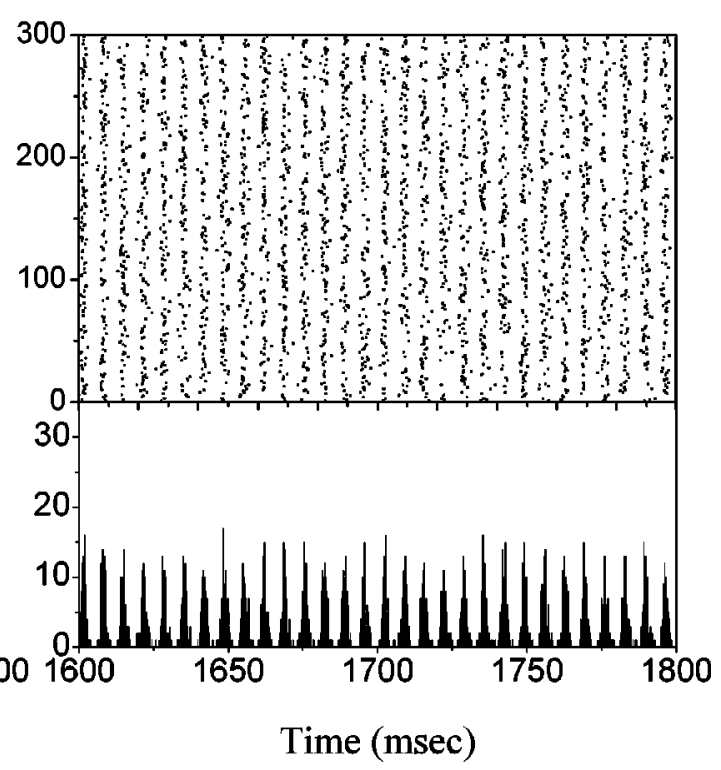

(d)

FIG. 2. Four sets of rastergram (upper panel) and global firing histogram (lower panel) with different coupling strengths $J$. (a) $J=0$. (b) $J_{e x}=10$. (c) $J_{e x}=10$ with a transmission delay of $7 \mathrm{msec}$ between neurons. (d) $J_{i n}=30$. In all cases, the constant bias $I=6 \mu \mathrm{A} / \mathrm{cm}^{2}$, the noise intensity $D=4$, and the number of neurons $N=300$. The time bin of the histogram is $0.2 \mathrm{msec}$.

limit cycle of coherence resonance. When a strong excitatory coupling exists [Fig. 4(b)], a sharp peak is seen at the CR frequency, which also corresponds to the global period of about $16-17 \mathrm{msec}$ found in Fig. 2(b). When a transmission delay of $7 \mathrm{msec}$ is added [Fig. 4(c)], the power spectrum shows three peaks. Apart from the two CR peaks at about 60 $\mathrm{Hz}$ and $120 \mathrm{~Hz}$, there is another sharp peak at about $110 \mathrm{~Hz}$ (period equals about $9 \mathrm{msec}$ ), which corresponds to the global period in Fig. 2(c), rather than the transmission delay. Finally, in the presence of strong inhibitory coupling [Fig. 4(d)], a sharp peak located at about $145 \mathrm{~Hz}$ (period equals about $7 \mathrm{msec}$ ) is seen apart from the CR peaks, which corresponds to the global period in Fig. 2(d), but is also close to the intrinsic delay.

In fact, there is an important difference between the intrinsic delay and external delay (either transmissional or synaptic). The effect of an external delay is merely to add a phase difference between the neurons. The global period is determined by the dynamics of the whole network. Nevertheless, the effect of postinhibitory rebound is not merely a delay. When a neuron undergoes a PIR process, its state is also under the refractory period which is insensitive to exter- 


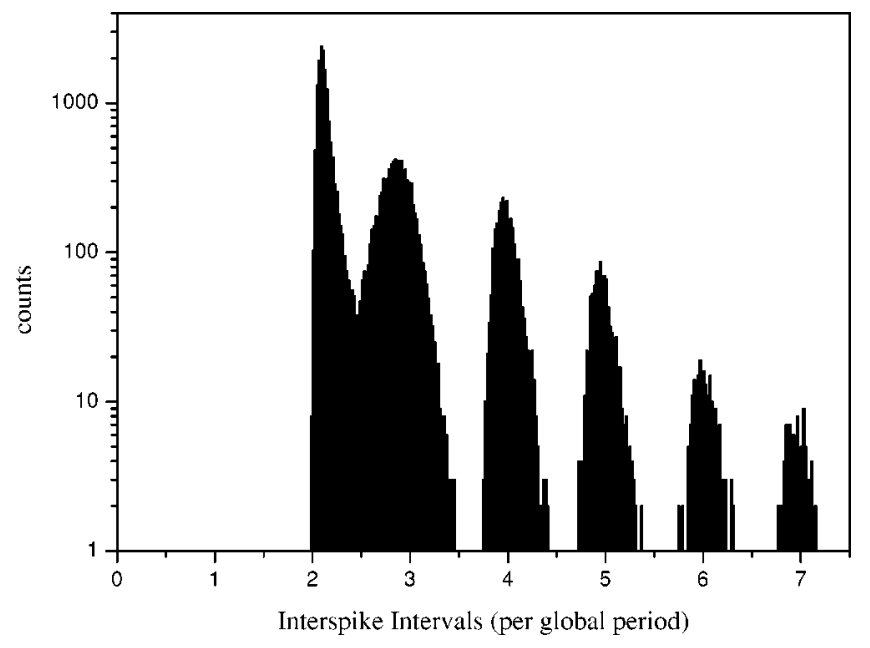

FIG. 3. Interspike interval histogram of one of the neurons in the network described in Fig. 2(d) $\left(J_{i n}=30, I=6 \mu \mathrm{A} / \mathrm{cm}^{2}, D\right.$ $=4, N=300$ ). The unit of the $x$ axis is the average global period calculated from a long time series of rastergram.

nal stimuli. After that, one spike is produced and then the neuron returns to the normal state. This extra refractory time scale (the PIR time scale) competes with the CR time scale. The shorter time scale is always chosen in the rastergram (by the definition of weak synchronization, the shortest observable period is chosen as the global period). This explains why the global period is closely related to the intrinsic delay time scale in this inhibitory network. In addition, since PIR is a one-off process that ends after the rebounded spike is fired, the continuous noise input must also exist for providing a continuous spike train through $\mathrm{CR}$, which brings about a continuous series of PIR events for making up the rastergram.
Apart from the necessity of large $J$ for the occurrence of synchronization (either strong or weak), there are also restrictions on the noise intensity $D$ and the number of neurons in the network $N$. In the excitatory case, there is a bellshaped curve for the measure of coherence [19], showing that an optimal range of $D$ and a large $J$ is necessary for maximum coherence or synchronization. Here we intend to study how $D$ and $J$ affect the degree of synchronization in an inhibitory network. Instead of using the measure of coherence, we introduce a new measure of synchronization $Y$. First, we divide the global firing histogram [lower panels of Figs. 2(a-d), for example] into many time windows of 100 msec each. Although the CR frequency varies with both $D$ and $J$, the time period is always smaller than $100 \mathrm{msec}$. For each time window, we can find a maximum height of firing counts at a certain time bin. By averaging over many time windows, we obtain an average maximum height $H_{\max }$. We then define $Y=\left(H_{\max }-H_{0}\right) / H_{\max }$, where $H_{0}$ is the average maximum height for $J=0$, and both $H_{\max }$ and $H_{0}$ are calculated from the corresponding histograms with the same conditions (bin size, etc.) and the same firing rate. This new measure may describe the strength of synchronization for a general case $J$. When there is no synchronization, $H_{\max }$ $\sim H_{0}$ and $\mathrm{Y} \rightarrow 0$. On the contrary, when synchronization occurs, $H_{\max } \gg H_{0}$, so that $\Upsilon \rightarrow 1$. $Y$ is a reliable measure of the synchronizing effect of coupling against the diffusion effect of noise, and the calculation method is applicable to both strong and weak synchronization. In Ref. [19], the measure of coherence is calculated from the power spectrum of one of the neurons in the network. Here the measure of synchronization is determined from the global firing histogram. Therefore our attention is paid to the overall behavior rather than the individual one.

In the main panel of Fig. 5(a), we plot $Y$ against $D$ for various $J_{i n}$ with $N=100$. For a certain $J_{i n}$, when $D$ is very
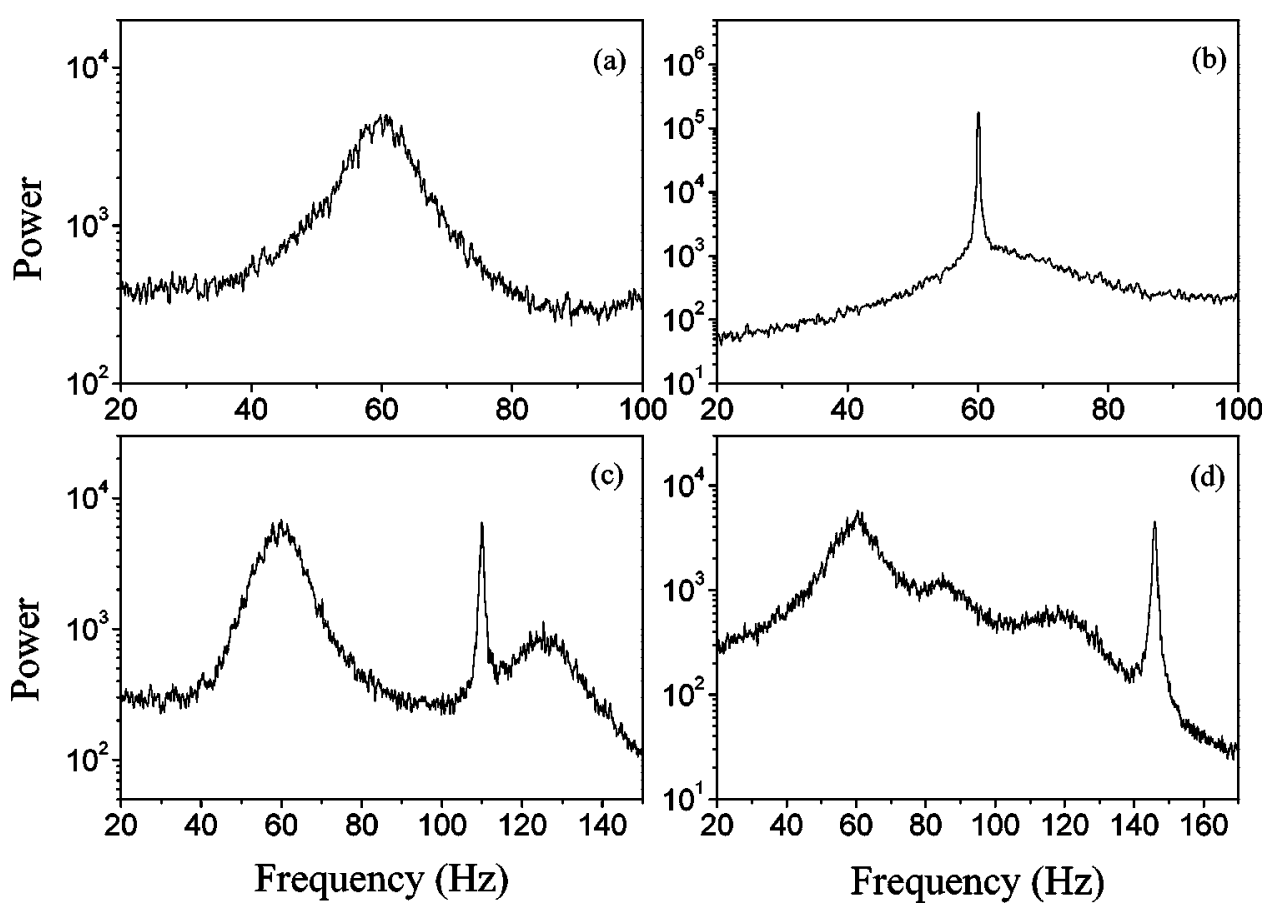

FIG. 4. Four sets of power spectrum of one of the neurons in the network of (a) $J=0$, (b) $J_{e x}$ $=10$, (c) $J_{e x}=10$ with a transmission delay of $7 \mathrm{msec}$ between neurons, and (d) $J_{i n}=30$. In all cases, the constant bias $I=6 \mu \mathrm{A} / \mathrm{cm}^{2}$, the noise intensity $D=4$, and the number of neurons $N=300$. 

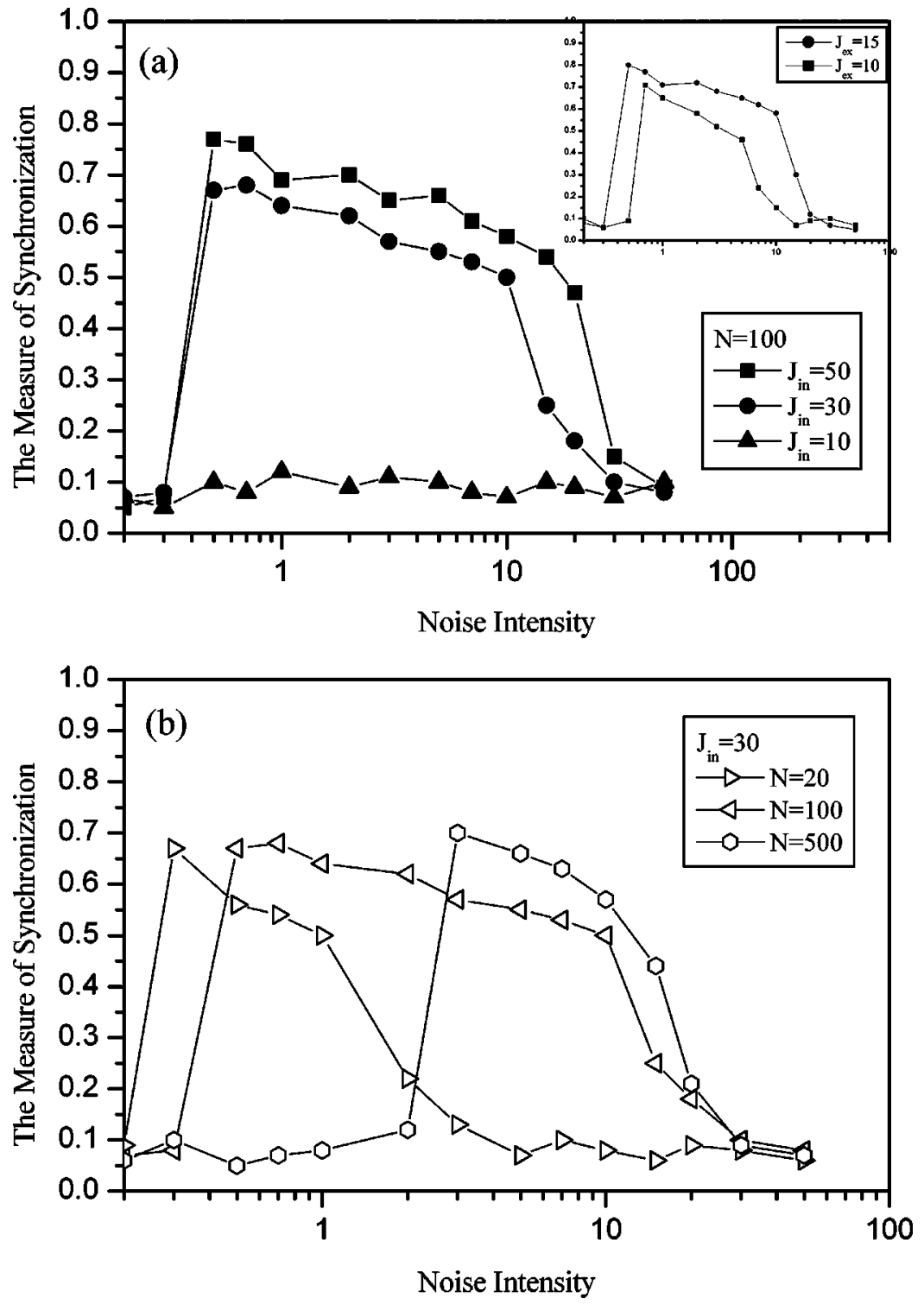

FIG. 5. The measure of synchronization $Y$ vs the intensity of noise $D$ for (a) different coupling strengths $J_{i n}=10,30,50$, with $N=100$; inset: $J_{e x}=10,15$ with $N=100$; (b) different network sizes $N=20,100,500$, with $J_{i n}=30$. In all cases, $I=6 \mu \mathrm{A} / \mathrm{cm}^{2}$. small, the firing is sparse and no synchronization can form. At the other extreme, when $D$ is very large, the firing pattern will be a mess. Therefore we can see a bell-shaped curve showing that the degree of synchronization is significant when the noise intensity $D$ is inside an optimal range from about 0.5 to 10 . If the coupling is not strong enough (for example, $J_{i n}=10$ ), there will be no synchronization for any $D$. For the excitatory coupling, similar bell-shaped curves can also be seen (inset).

The dependence of synchronization on the number of neurons $(N)$ of the network can be seen in Fig. 5(b), where we plot $Y$ against $D$ for various $N$, with $J_{i n}=30$. We found that for a larger $N$, the optimal range of $D$ shifts to a higher value. This is due to the constant bias being set close to $I_{C}$ but far away from $I_{C}^{\prime}$. On the one hand, a small input is sufficient to cross the threshold $I_{C}$ to generate a spike through CR. On the other hand, a large input is necessary to overcome the rebound threshold $I_{C}^{\prime}$ to generate the PIR spike. We know that it is the PIR spikes that organize the neurons because they are induced by the inhibitory coupling. Since the coupling strength is averaged by $N-1$ pairs of neurons, when one neuron fires, the synaptic current received by other neurons is relatively smaller if $N$ is large. Therefore a larger $D$ is required to compliment for the weakened synaptic input for large $N$, and vice versa. In addition, as the contribution of individual behavior becomes less significant, a larger network is also more stable and is capable of tolerating even higher $D$. The result is that the optimal range of $D$ shifts upward. Interestingly, an optimal network size $N=100$ can be found where the optimal noise range is broadest (in log scale).

To conclude, we have identified the effects of the coupling strength $J$, the noise intensity $D$, and the network size $N$ on the occurrence of strong and weak synchronization. In particular, we found an intrinsic delay in a strongly inhibitory network, which has an important contribution to the weak synchronization. We wish to remark that synchronization and periodic oscillations are not necessary to occur together. There can be nonperiodic synchronization or nonsynchronous periodic oscillators. In the present study, both 
synchronization and periodic oscillation occur. The effect of $\mathrm{CR}$ is to provide a continuous and nearly periodic spike train, but the effect of PIR is twofold: to induce synchronization by providing feedbacks to the system, and to prevail over the CR time scale in determining the global period. In addition, the PIR delay is found to be sensitive to the coupling strength and can be optimized by the size of the network group.

We would like to thank Dr. Yuqing Wang for his useful suggestions and comments.
[1] D. Hansel and H. Sompolinsky, Phys. Rev. Lett. 68, 718 (1992); S.R. Campbell and D.L. Wang, Physica D 111, 151 (1998); D. Terman, N. Kopell, and A. Bose, Physica D 117, 241 (1998); M.Y. Choi, H.J. Kim, D. Kim, and H. Hong, Phys. Rev. E 61, 371 (2000); L. Neltner and D. Hansel, Neural Comput. 13, 765 (2001); Y.D. Sato and M. Shiino, Phys. Rev. E 66, 041903 (2002).

[2] N. Kopell, in Neural Control of Rhythmic Movements in Vertebrates, edited by A.H. Cohen, S. Rossignol, and S. Grillner (Wiley, New York, 1987), p. 369; D. Hansel, G. Mato, and C. Meunier, Phys. Rev. E 48, 3470 (1993); D. Golomb and J. Rinzel, Physica D 72, 259 (1994); Y. Wang, Z.D. Wang, Y.X. Li, and X. Pei, J. Phys. Soc. Jpn. 72, 443 (2003).

[3] W. Singer and C.M. Gray, Annu. Rev. Neurosci. 18, 555 (1995)

[4] R. Llinas and U. Ribary, Proc. Natl. Acad. Sci. U.S.A. 90, 2078 (1993).

[5] M. Steriade, D.A. McCormick, and T.J. Sejnowski, Science 262, 679 (1993); M.A. Whittington, R.D. Traub, and J.G.R. Jeffreys, Nature (London) 373, 612 (1995).

[6] R.C. O'Reilly and Y. Munakata, Computational Explorations in Cognitive Neuroscience (MIT Press, Cambridge, 2000), p. 105.

[7] U. Ernst, K. Pawelzik, and T. Geisel, Phys. Rev. Lett. 74, 1570 (1995).

[8] W. Rall, J. Neurophysiol. 30, 1138 (1967); W. Rall, in Methods in Neuronal Modeling: from Synapses to Networks, edited by C. Koch and I. Segev (MIT Press, Cambridge, 1989), p. 9.

[9] S. Kunec and A. Bose, Phys. Rev. E 63, 021908 (2001).

[10] R.C. Foehring and A.R. Wyler, Neurosci. Lett. 110, 279 (1990); L.R. Silva, Y. Amitai, and B.W. Connors, Science 251, 432 (1991).

[11] A.L. Hodgkin and A.F. Huxley, J. Physiol. (London) 117, 500 (1952)

[12] X.J. Wang and J. Rinzel, Neural Comput. 4, 84 (1992); X.J.
Wang and J. Rinzel, Neurosci. Lett. 53, 899 (1993).

[13] S. Kuwada and R. Batra, J. Neurosci. 19, 2273 (1999); E.W. Large and J.D. Crawford, J. Comput. Neurosci. 13, 125 (2002).

[14] R.A. Satterlie, Science 229, 402 (1985); E.A. Arbas and R.L. Calabrese, J. Neurosci. 7, 3953 (1987).

[15] P.V.E. McClintock and F. Moss, in Noise in Nonlinear Dynamical Systems, edited by F. Moss and P.V.E. McClintock (Cambridge University Press, Cambridge, England, 1989), Vol. 243.

[16] This minimun delay is not found in the following simulations when the input is a noise and the total synaptic current from the network to single neuron varies from time to time.

[17] G. Hu, T. Ditzinger, C.N. Ning and H. Haken, Phys. Rev. Lett. 71, 807 (1993); W.J. Rappel and S.H. Strogatz, Phys. Rev. E 50, 3249 (1994); A. Longtin, ibid. 55, 868 (1997); A.S. Pikovsky and J. Kurths, Phys. Rev. Lett. 78, 775 (1997); J.R. Pradines, G.V. Osipov, and J.J. Collins, Phys. Rev. E 60, 6407 (1999).

[18] S.G. Lee, A. Neiman, and S. Kim, Phys. Rev. E 57, 3292 (1998).

[19] Y. Wang, D.T.W. Chik, and Z.D. Wang, Phys. Rev. E 61, 740 (2000).

[20] This result is different from that in P.H.E. Tiesinga and J.V. Jose, Neurocomputing 32-33, 249 (2000); X.J. Wang and J. Rinzel, Neural Comput. 4, 84 (1992). Their network models are autonomous systems where only an initial clamp is needed to provide a sustained oscillation. The global oscillation is generated purely by PIR ith an optimal tuning of synaptic delay, and the neuron model itself is not necessarily an oscillator. Therefore the frequencies of strong and weak synchronization are the same in their models. In our model, however, there are two different time scales: CR and PIR. In the presence of a continuous noise input and the absence of synaptic delay, the global frequency is determined by the interplay of both time scales. 